\title{
La adaptación de la forma a las cargas en el arte estructural: desde las pilas y el arco, a la torre
}

\section{Adaptation of the shape to loads on structural art: from piles and arch, to the tower}

\author{
$\underline{\text { R. Serrano-López }}^{(*)}$, J. Mínguez-Algarra ${ }^{(*)}$, F. Cambronero-Barrientos ${ }^{(*)}$, D. Saldaña-Arce ${ }^{(*)}$
}

\section{RESUMEN}

La forma de la Torre Eiffel ha sido varias veces analizada, buscando relaciones físicas y matemáticas. Este artículo pretende abordar este análisis desde un punto de vista diferente, la evolución histórica y secuencial del diseño a lo largo de la trayectoria de la empresa de Eiffel. De esta manera, puede comprobarse la relación entre la experiencia acumulada en diferentes obras y técnicos. Se intenta además atribuir los méritos que corresponden a todos los colaboradores de Eiffel a lo largo de esos años. En definitiva, se contrasta y corrobora de esta manera a la solución final de la Torre como un magnífico ejemplo de arte estructural, como ya ha sido descrito en otras ocasiones.

Palabras clave: Arte estructural; pila; puente arco; Torre Eiffel; estética.

\section{ABSTRACT}

The shape of the Eiffel Tower has been discussed several times, seeking for physical and mathematical relationships. This article aims to address this analysis from a different point of view: the historical and sequential evolution of design over the course of the Eiffel company. Thus, the relationship between the experience gained in different works and technicians can be verified. The paper also tries to give merit to work of all the collaborators of Eiffel over those years. In short, the final solution of the Tower is contrasted and corroborated as a great example of structural art, as has been described in the past.

Keywords: Structural art; pile; arch bridge; Eiffel Tower; aesthetics.

(*) Universidad de Burgos (España)

Persona de contacto/Corresponding Author: robertosl@ubu.es (R. Serrano-López)

Cómo citar este artículo/Citation: Serrano-López, R., Mínguez-Algarra, J., Cambronero-Barrientos, F., Saldaña-Arce, D. (2014). La adaptación de la forma a las cargas en el arte estructural: desde las pilas y el arco, a la torre. Informes de la Construcción, 66(534): e021, doi: http://dx.doi.org/10.3989/ic.13.005.

Licencia/License: Salvo indicación contraria, todos los contenidos de la edición electrónica de Informes de la Construcción se distribuyen bajo una licencia de uso y distribución Creative Commons Reconocimiento no Comercial 3.o. España (cc-by-nc). 


\section{INTRODUCCIÓN}

\subsection{Los contornos de las estructuras y la estética del conjunto}

Los trabajos de Alan Holgate (1), Bill Addis (2) y, sobre todo, de David Billington (3) (4) (5) suelen ser reconocidos como las referencias sobre el concepto de «arte estructural». Tal como ha sido revisado en otras ocasiones (6), bajo este término se describe a las obras que han incluido en el proceso de diseño (en la evolución de la forma hasta su geometría definitiva) el desarrollo de las características propias del material: desde elementos sencillos (en los cuales la esbeltez o la delgadez aparece como denominador común), hacia su combinación de manera integrada y su relación con el entorno en el que se ubica (en forma de contraste y/o armonía). Billington, que basó sus estudios en la comparación crítica de los diseños de estructuras desde los comienzos de la ingeniería civil como profesión, acota definitivamente el «arte estructural», el arte de los ingenieros, mediante las tres cualidades que debe de satisfacer y precisamente le diferencian de otras artes como la escultura o la arquitectura: eficiencia, economía y elegancia. Entre sus conclusiones (7), sugiere la necesidad de una elección consciente por parte del diseñador de una forma expresiva y estructuralmente lógica. Así, la eficiencia y economía exigidas a los ingenieros, deben equilibrarse con la elegancia fruto de la elección libre del proyectista para definir la solución definitiva.

Por otra parte, y de forma íntimamente relacionada con el concepto anterior, debe distinguirse el término «honestidad estructural», que puede envolver otros más específicos como «honestidad funcional» $\mathrm{y}$ «honestidad del material». Este concepto ha sido utilizado en bibliografía de crítica arquitectónica así como de estética de la construcción civil. Sus principios teóricos fueron argumentados por Ruskin en 1849 (8); posteriormente Adolf Loos (9), plasmó de manera radical en sus diseños estas hipótesis, criticando duramente el uso de la decoración o el añadido como recurso arquitectónico, si bien el funcionalismo de sus propuestas relegaba la dimensión estructural a un segundo plano. El profesor Aguiló (10) destacó cómo los teóricos del movimiento moderno (Pevsner, Giedion o Behrendt) fueron en cierto modo culpables de la identificación directa de la ingeniería con la técnica; si bien permitió la salida de la «falacia» historicista de finales del siglo XIX, una visión meticulosamente sesgada de algunos de estos conceptos permitió a ciertos técnicos proyectar estructuras como un resultado simplemente tecnológico, derivado del cálculo, extrapolando hasta el extremo las ideas racionalistas.

La «honestidad estructural», sin embargo, pretende asegurar para cada material a su posición más adecuada, sin enmascararse. Las partes superfluas o sobreabundantes desaparecen, proporcionando de esta manera las formas más puras correspondientes a las hipótesis de carga previstas. De manera sucinta, puede definirse como la adaptación de las formas de las estructuras a las acciones que debe de soportar durante su vida útil; durante el proceso de maduración creativa, la naturaleza y propiedades del material de construcción, junto a la función para el que se proyecta la estructura, adoptan una importancia integral en la evolución del propio diseño y, consecuentemente, en la manera de resistir y transmitir las cargas hacia el terreno. En este sentido, Ove Arup defiendía (11), como puso de manifiesto en sus conferencias en Dublín y Londres de 1954, que la «firmeza» de la estructura (en rela- ción a la estabilidad y durabilidad) no es sino una condición necesaria para conseguir la comodidad y el deleite estético, quedando éstas subordinadas a la primera; describía a la estructura como un medio para conseguir el todo del diseño, pero que debe de tenerse en cuenta desde los primeros estudios de alternativas para asegurar la sencillez y economía de la solución final.

Respecto a la arquitectura, la obra de algunos autores ha estado ligada precisamente a este tipo de consideraciones durante todo su proceso creativo. Quizá el caso de las formas poligonales y curvas sea el que permita una compresión más clara y directa de estos conceptos, como ocurre con Gaudí o Candela (12) (13) (14). Por otro lado, las grandes estructuras se caracterizan por su monumentalidad, su visibilidad desde largas distancias. El tamaño y la escala son variables importantes en la percepción. Este es el caso de las grandes obras civiles, en las cuales la forma de su contorno sobresale respecto a los detalles.

El caso de la torre Eiffel (Figura 1) ha sido analizado en otras ocasiones y por diferentes autores desde el punto de vista matemático, relacionando su forma con una ecuación de ajuste (15) (16). En estos trabajos, se partía de ciertas hipótesis de equilibrio y/o propiedades del material. Otros puntos de vista han revisado la jerarquía de la composición (17), o el perfil óptimo del contorno (18). Este artículo se aleja de las propuestas anteriores, realizando una retrospectiva entorno al

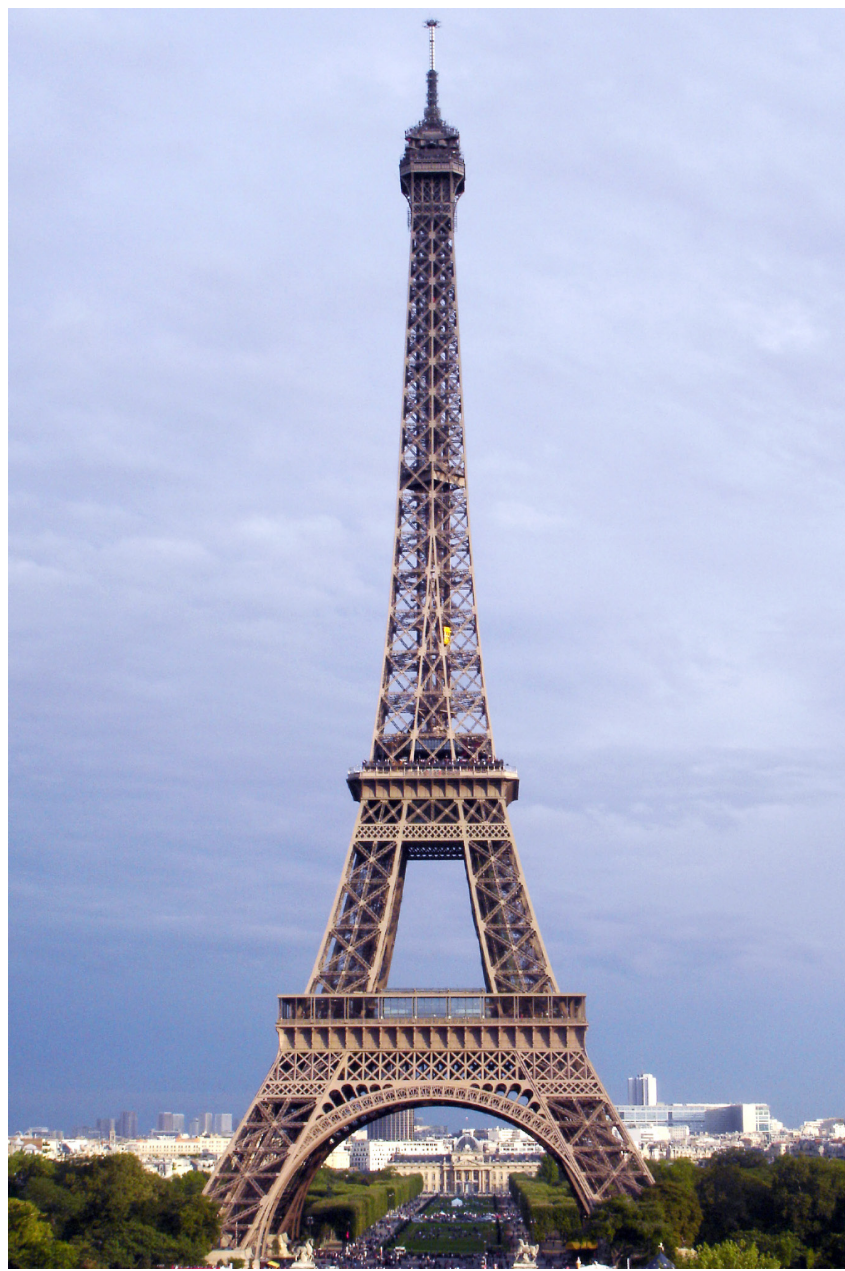

Figura 1. Torre Eiffel (París, 1889, fotografía de los autores). Una de las estructuras más famosas del mundo. 
análisis cualitativo de las formas externas de las construcciones metálicas, y relacionándolas con su eficiencia estructural. Para ello se revisa su evolución durante el siglo XIX, ligadas directamente con la estética de la obra completa y su percepción a diferente escala. Varias de las obras de la constructora G. Eiffel et Cíe constituyen claros ejemplo del dominio de la forma y su uso intencionado hacia la emoción estética: auténtico arte estructural.

\subsection{La idea de la torre}

Quizá la forma de la Torre Eiffel de París sea uno de los diseños más fáciles de reconocer y, además, de asociar como icono a una determinada ciudad. Todo un logro de sus creadores. Esta obra metálica fue concebida como un monumento que sirviera de entrada al complejo de la exposición Universal de 1889, y debía servir para celebrar el centenario de la Revolución Francesa. Durante este siglo, varias naciones proponían proyectos de torres de gran altura (19): Trevithick presenta una columna de fundición de 1000 pies en Inglaterra (para celebrar el Reform Bill de 1832); una torre observatorio de $90 \mathrm{~m}$ en la Exposición de Nueva York de 1853, ideada por James Bogardus; una torre de 1000 pies en la Exposición de Filadelfia de 1876 (proyecto de Clarke y Reeves); o incluso el Washington Monument, un obelisco de piedra que pretendía alcanzar los 183 metros de altura, aunque tuvo que reducirse a 169 metros debido a problemas durante la ejecución, inaugurándose en 1885 .

Las noticias de estas propuestas llamaron la atención a Sébillot, un ingeniero francés, que se propuso diseñar una torre a modo de faro. Se asoció para ello con el arquitecto Jules Bourdais, autor del parisino Trocadero, diseñando en 1881 una torre cilíndrica que recordaba a la de Pisa. Pero fueron dos ingenieros de la empresa de Eiffel (Noguier y Koechlin) los que, tras un intercambio de ideas en mayo de 1884 (20), comenzaron a plantearse seriamente el proyecto de una torre de gran envergadura para la Exposición que se celebraría cinco años después. De esa manera, Koechlin realiza los primeros cálculos generales y dibuja un esbozo de la forma que considera más apropiada: un gran pilono formado por cuatro barras curvas en celosía, separadas entre sí en la base y unidas en el vértice, conectadas en diferentes puntos por vigas horizontales. Las líneas maestras del diseño se habían decidido ya en junio de 1884 . Como se comprueba, el reto natural de alcanzar mayores dimensiones en sus trabajos fue una inspiración para los ingenieros del siglo XIX, que buscaron en la barrera de los $300 \mathrm{~m}$ (1000 pies) un hito para demostrar el avance de la técnica.

\section{UN BREVE APUNTE SOBRE SEYRIG Y EIFFEL}

Antes de abordar el proceso de evolución de las formas, merece la pena apuntar algunos detalles de las estas biografías. La manera de ser y trabajar de estos dos técnicos explica el devenir de cada una de sus vidas y de sus obras.

Existe numerosa bibliografía sobre el ingeniero francés Gustave Eiffel. El hecho de que su empresa constructora adoptara la misma denominación ha permitido pasar a la memoria colectiva universal. Nació en Dijon en 1832 en el seno de una familia acomodada (21). Tras ser rechazado por l'Ecôle Polytecnique de Paris, acaba por formarse como ingeniero en l'Ecole Centrale des Arts et Manufractures (1885) sin destacar como estudiante. Su primer trabajo lo consigue a través de un conocido de su madre, Charles Nepveu, contratista, constructor, y dueño de un taller industrial. Bajo su apadrinamiento entra en la Compañía de Ferrocarriles del Oeste, con lo que consigue presencia en las obras de un importante puente en Burdeos (en torno a 1860). Esta etapa marca el devenir posterior de Eiffel, establece nuevos contactos (los hermanos Pereire, Wilhelm Nördling) y aprende a manejar los tiempos de ejecución (19).

Tras diversos devenires, decide ponerse por su cuenta como ingeniero independiente el año 1865, dedicándose a la supervisión de ejecución, no al diseño. Se especializa en el control de tiempos y costes, logística, programación. Pronto le llegan numerosos encargos que le mueven a arrendar unos talleres de metal y establecerse definitivamente como empresario contratista (1867). En esta y otras ocasiones tuvo que solicitar financiación a su familia para continuar con sus negocios (19).

Su apellido se ha adueñado de la autoría de una gran cantidad de puentes y estructuras. En realidad Eiffel fue un brillante hombre de negocios. Su formación técnica y, sobre todo, su personalidad aventurera e innovadora le permitieron hacer crecer rápidamente la empresa que fundara. Consiguió establecer una fructuosa red de contactos, comenzando por las relaciones que surgían en los círculos republicanos y francmasones en que se movía (19) (22). Supo aprovechar el desarrollo de las redes ferroviarias en Francia, Europa y el resto del mundo para desarrollar y demostrar sus habilidades innatas. Tenía un talento natural para rodearse de los mejores especialistas y técnicos que podía encontrar en cada momento. Esta última fue, seguramente, la clave del éxito de la compañía Eiffel et Cíe. En el prólogo del libro que Lemoine dedica a su vida (19), los editores llegan a definir a Eiffel como «patrón de una empresa floreciente que se apropia de las ideas de sus colaboradores, pero también más hábil que ninguno en dar valor y consistencia a esas ideas; cabeza de una sociedad de construcción cuyos fines siempre serán lucrativos, pero también inventor que asume el riesgo de echarse a la espalda dicha sociedad para desarrollar un proyecto en el que cree», llegando a definir su personalidad como «tan obstinada como oportunista».

Durante el primer año (1867) Eiffel ya consigue los contratos de dos grandes viaductos, Rouzat y Neuvial, gracias a su conexión con Nördling (23), y ya acumulaba otros anteriores para la Exposición Universal y la Galería de Bellas Artes (20).

Por otra parte poco se ha escrito sobre la biografía de Teóphile Seyrig. El profesor Manuel Azeredo, de la Universidad de Oporto, ha recopilado algunos datos a través del Centre de Documentation de l'Ecole Centrale de Paris, y pueden encontrarse algunos más en ciertas publicaciones francesas y españolas.

Fue un ingeniero de gran talento de origen alemán. Nacido en Berlín, fue número uno de su promoción en l'École Centrale. Mientras su padre vivía en Bruselas, ingresó de forma brillante en l' Ecole en agosto de 1861, consiguiendo en tres años la especialidad en mecánica. Tras acabar sus estudios, permanecerá en París casi toda su vida, coordinando desde allí sus trabajos. Procedía de familia adinerada, y fue captado como socio capitalista mayoritario y cofundador de la empresa de Gustave Eiffel, a la que perteneció entre el 6 de octubre 
de 1868 hasta el 30 de junio de 1879 (20). La habilidad de Eiffel en la negociación y su experiencia se hacen patentes en el propio contrato establecido entre ambos. Seyrig aporta más capital y su capacidad técnica, mientras Eiffel se pone al mando de la compañía como gerente único, dueño de los talleres y gestor de sus clientes (19).

Seyrig trabaja durante once años como técnico y calculista de esta empresa, quizá los años más importantes para la misma: la ampliación de capital permite afrontar obras de mayor envergadura y abrir nuevos mercados. Dentro de sus trabajos mientras estaba asociado con Eiffel, pueden destacarse entre otras obras: la estación de Pest (1877), sobre todo el Puente de María Pía sobre el Duero en Oporto (1877) (Figura 2), y el Puente de Viana do Castelo (1878).

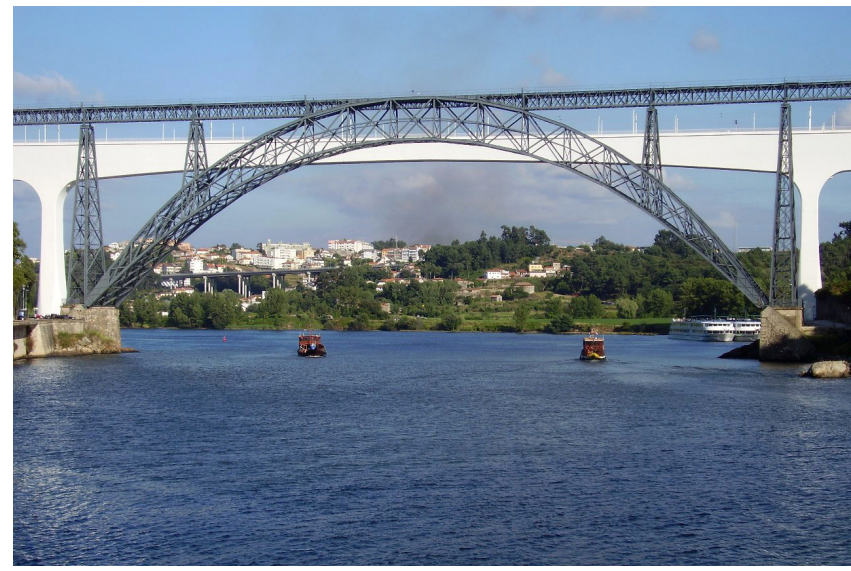

Figura 2. Puente de Maria Pia (Oporto, 1877, fotografía de los autores). La estructura fue diseñada por Teóphile Seyrig y construido por Eiffel et Cíe.

A partir de 1879 Seyrig continua con la sociedad belga Willebroek, y finalmente crea su propia compañía constructora. De esta segunda etapa, sin Eiffel, debe mencionarse el sobresaliente Puente de Luis I, también en Oporto (1885) (Figura 3). También colaboró con Henri de Dion en la concepción del Vestíbulo de entrada a la exposición universal de París de 1878 (19) (24). En 1879 recibe el premio anual de la Sociedad de Ingenieros Civiles de Francia de por su trabajo Mémoire sur le Pont construit sur le Douro par M. G. Eiffel (25).

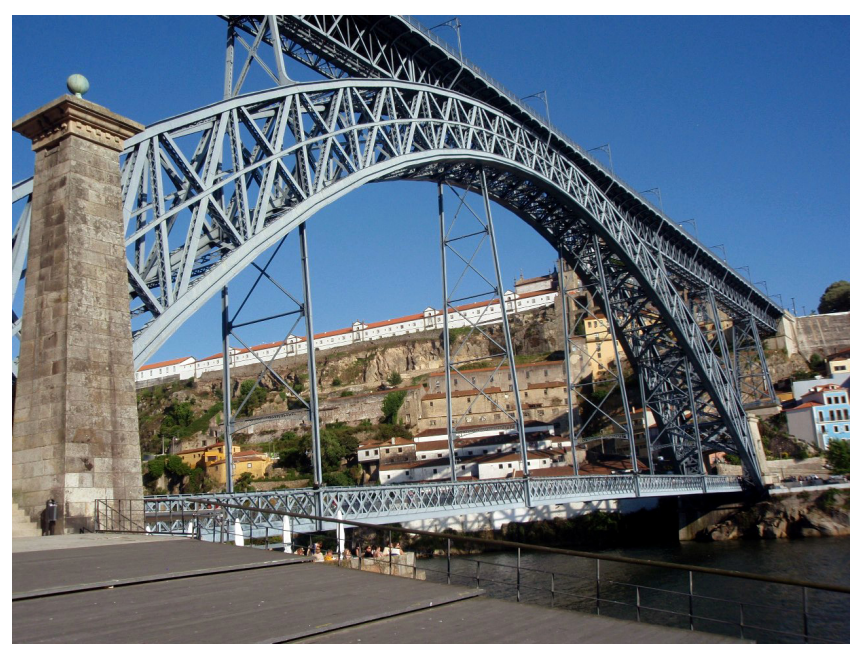

Figura 3. Puente Luis I (Oporto, 1885, fotografía de los autores). Un ejemplo temprano de la forma arco-tímpano, obra de T. Seyrig.
Pueden señalarse otros datos poco conocidos sobre la vida de Seyrig. En 1891, se abre la explotación de un funicular en París, de vía sencilla y ancho métrico, que proyectó en colaboración con el también ingeniero Mr. Bienvenu (26). También existen noticias de un proyecto no ejecutado para un nuevo puente sobre el río Tajo en Lisboa en el año 1889, desde la Roca del Conde Obidos hasta Almada, colaborando con Edmond Bartissol (27). Éste consistía en una colosal solución metálica de más de $2 \mathrm{~km}$ de longitud, tanto para vehículos como ferrocarriles, permitiendo el paso bajo su tablero del más grande de los barcos. Su elevado presupuesto impidió que el pueblo lisboeta pudiera disfrutar de una solución para el cruce del Tajo hasta 1967. En España, diseña y construye los puentes de la línea Valladolid-Ariza, y su empresa constructora fue la encargada de erigir el primer puente metálico sobre el Duero en Zamora (1891) (28), en la línea PlasenciaAstorga, y cuyos tramos fueron sustituidos el año 1933 (29).

Seyrig formó parte del comité de redacción de la revista «Le Génie Civil», y fue colaborador habitual de la Sociedad de Ingenieros Civiles. Fue también miembro (desde 1881) de la Association francaise pour l'avancement des sciences (30). Escribió varios artículos y reseñas de índole técnica a lo largo de su vida, destacando la traducción al francés del libro Éléments de Statique Graphique appliquée aux constructions, de Müller-Breslau (Librairie Polytechnique Baudry et Cie, Éditeurs- Paris 1886).

Además de su faceta técnica, se le reconocen sus inquietudes hacia la arqueología, astronomía y psicología (31), siendo autor y traductor de varios textos de estas disciplinas. En definitiva, sus intereses intelectuales y su manera de entender la vida eran algo diferentes a los de Eiffel.

\section{UNA NUEVA VISIÓN: LA PERSPECTIVA HISTÓRICA DE LA FORMA}

\subsection{Pila de puente}

La Torre Eiffel no es sino una gran pila de puente, ampliando su tamaño y cambiando sus funciones. Para el objetivo perseguido, puede hacerse un repaso a las soluciones propuestas para esta parte de los viaductos durante el siglo XIX, utilizándose para ello los ejemplos en los que hubo intervención de Eiffel o sus colaboradores.

El primer uso de las celosías estructurales se suele ubicar en las estructuras de madera de los ferrocarriles norteamericanos durante todo el siglo XIX (Figura 4). Los valles se salvaban mediante pilas de gran anchura cuya sección en planta aumentaba hacia el terreno, a veces formando una palizada continua en toda la longitud de los puentes (32) (33). La variación de ancho fue utilizada también en pilas de construcción tradicional, en piedra, de algunos puentes de vigas metálicas (incluso durante el siglo XX). Aumentando la superficie de estas pilas, se buscaba un reparto de las cargas verticales en los apoyos inferiores.

La generalización del uso del metal trajo consigo las primeras experiencias de su uso en pilas (Figura 5). En 1856 se construye el puente sobre el río Sitter de Bruggen (Suiza) (33). Las pilas fueron ejecutadas con tubos de fundición unidos por barras que formaban una red ortogonal. Un año más tarde se construye el puente de Crumlin (Gales), conocido por ser el primero en utilizarse la viga Warren. Sus pilas, de hasta 


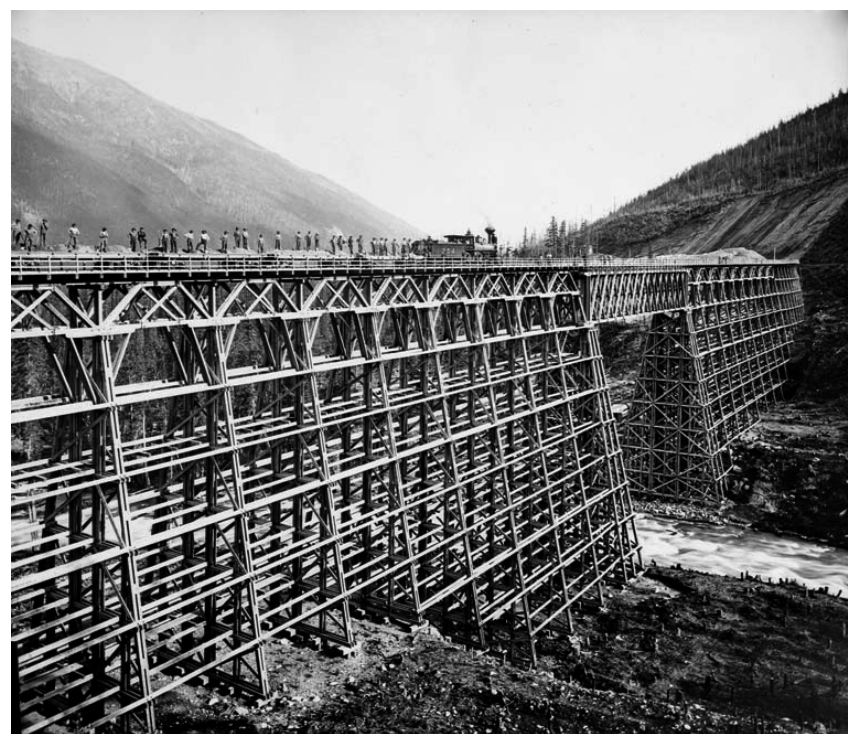

Figura 4. Mountain Creek bridge (British Columbia, Canadá, 1880-1890, fotografía de Norman Denley recogida en Library and Archives Canada / PA-066576).

$64 \mathrm{~m}$ de altura, estaban formadas por 14 tubos verticales de fundición solidarizados entre sí por un sistema de piezas horizontales y diagonales (32).

En 1862, Wilhelm Nördling interviene junto a Etzel en el viaducto de Grandfey (Suiza). Sus enormes pilas, de hasta 76 metros, se ejecutan con tubos conectados mediante una tupida celosía similar a la de las vigas Town que soportaban. Nördling se convierte en un especialista en viaductos ferroviarios. En 1864, construye con Felix Moreaux el viaducto de Besseau-sur-Creuse (33) ejecutando cada pila desde la propia viga en voladizo.

Uno de los saltos cualitativos en la técnica de las pilas surge con la construcción de los viaductos de la línea Commentry-Gannat (1867-1869). Con un nuevo diseño atribuible al propio Nördling, según describió años antes en los Annales de Ponts et Chaussées, se reducía el número de tubos hasta cuatro (uno por cada esquina) (34). Para compensar la disminución de rigidez frente a cargas transversales, debido al aumento de la esbeltez, se aumentaba la sección en la base.
Se hacía progresivamente mayor hasta llegar al tamaño del apoyo, adoptando una delicada forma curva. La unión entre los tubos se realiza mediante diagonales.

Los cuatro puentes de esta línea (Rouzat, Bouble, Bellon y Neuvial) adoptan soluciones muy similares, aunque fueron ejecutados por diferentes constructoras, con Nördling al mando de todas las construcciones. De entre todos ellos, las pilas con mayores diferencias son las de Bellon (la base es rectangular, en vez de elíptica como los otros tres). A Eiffel le adjudican los de Rouzat y Neuvial, en los cuales son las vigas los elementos diferenciadores (33).

Por tanto, debemos atribuir a Wilhelm Nördling la primera referencia de un diseño de pilas abocinadas en la base. Algunos autores siguen otorgando a Eiffel esta contribución (35), pero es necesario empezar a reconocer los a los auténticos creadores y no sólo a alguno de los constructores. También hay que recordar que, al menos durante unos meses, Téophile Seyrig también se va familiarizando con estas formas curvas y a su explicación técnica. Su relación profesional con Eiffel comenzó en octubre de 1868, y aprovechará estas experiencias en trabajos posteriores en los que el efecto del viento transversal es una variable importante en el cálculo.

Nuevos ejemplos menos conocidos de esta tipología de pilas se encuentran en Portugal. El Centro Português de Fotografia conserva imágenes de dos puentes (sobre el año 1877), en la línea Caminho de Ferro do Douro, y denominados Viadutos da Palla y das Quebradas. Lemoine recoge el primero de ellos llamando la atención sobre su parecido al de Rouzat (19). De hecho, el Viaduto de Vila Meã y el Ponte do Tâmega, en la misma línea de ferrocarril, se han atribuido a la compañía de Eiffel (19) (36) (37).

\subsection{El arco}

El Puente de Maria Pia en Oporto (Figura 2) es una obra especial entre las ejecutadas por la compañía Eiffel et Cíe. El profesor David Billington ha destacado esta obra en numerosas ocasiones como uno de los mejores ejemplos de arte estructural en la ingeniería civil (3) (38) (39). También en este caso el sello de la constructora ha ocultado en cierta forma al ingeniero que concibió su especial forma. No obstante, el debate sobre la autoría ha sido revisado en varias ocasiones (37) a)

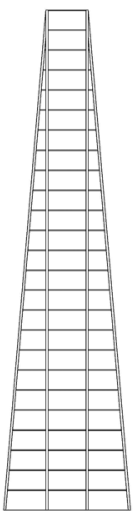

1856 b)

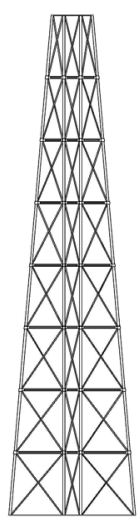

1857 c)

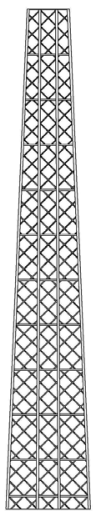

1862 d)

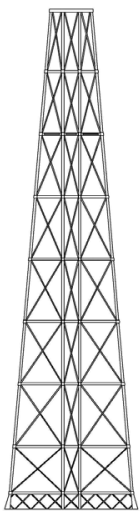

1864 e)

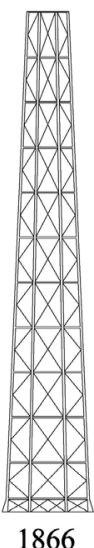

f)

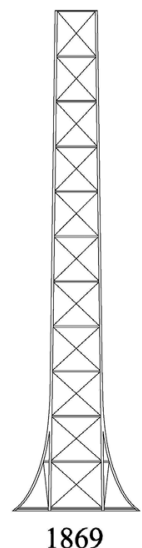

g)

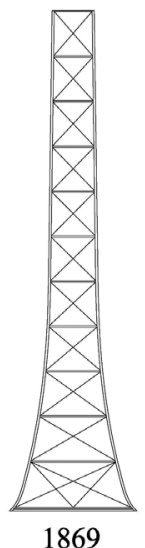

Figura 5. Secuencia evolutiva de la forma y estructura de las pilas. Esquemas de algunas pilas de viaductos: a) Bruggen; b) Crumlin; c) Grandfrey; d) Busseau-sur-Creuse; e) Cère; f) Bouble, Neuvial, Rouzat; g) Bellon. 
(40) otorgando a Seyrig su diseño inicial. De hecho, fue él el que describió su trabajo en 1878 a la Societé des Ingénieurs Civil. Y la propia empresa redactó una reseña para acompañar la maqueta mostrada en la Exposición Universal de ese mismo año, señalando que los cálculos del proyecto fueron efectuados por dos colaboradores de Gustave Eiffel: Téophile Seyrig y Henri de Dion (40).

El puente es resultado de un adecuado y riguroso ajuste de las formas en relación a los esfuerzos que tiene que soportar. Presenta, en alzado, una doble articulación. Mientras, en planta, fue calculado con doble empotramiento. El efecto del viento provoca que, tras el recálculo, su contorno tridimensional describa una doble curvatura. Seyrig dotó de mayor sección aquellas posiciones que lo exigían y en las direcciones en que era necesario, en coherencia con las hipótesis de sustentación. La solución final se fusiona con la propia jerarquía estructural, y consigue que el arco metálico adquiera una personalidad total al contemplarlo desde una cierta distancia (Figura 2). La honestidad estructural es quizá la más importante de sus características, y la estética de su conjunto se suma a la del excepcional paisaje en que se enmarca.

El ajuste parabólico de la planta ya no es un simple abocinado como en las pilas de Nördling. Es el efecto de adaptar la forma a la necesidad, que deriva estrictamente del cálculo y las particularidades constructivas sin añadidos o accesorios. Las líneas del contorno, cordones superior e inferior, destacan sobre la ligereza de la celosía interior, que tan sólo se complica en las zonas que así lo requiere. La transparencia facilita el paso del aire, minimizando la superficie expuesta.

En su ejecución (entre 1876 y 1877) intervinieron dos nuevos fichajes de Eiffel: Émile Noguier, ingeniero de minas, y Jean Compagnon, jefe de montaje (19). El valor de la obra fue reconocido rápidamente, proponiéndose incluso un proyecto similar para un pabellón-puente en la Exposición Universal de 1878. Debido al éxito de este diseño, Eiffel utilizó el mismo concepto para presentar una propuesta para un puente sobre el río Truyère en Francia (Viaducto de Garabit) con un aspecto muy similar. La adjudicación de éste el 14 de junio de 1879 , sin pasar por un concurso previo, provoca que Seyrig se enfrente a Eiffel: no consideraba justa la manera de aprovechar sus ideas sin repartir los beneficios que pudieran surgir a partir de éstas. El desacuerdo acaba con la ruptura del contrato el 30 de junio de ese mismo año (19).

Eiffel consigue rápidamente un nuevo técnico para sustituirle. Se trata de Maurice Koechlin, alumno recomendado por Karl Culmann (41), que es contratado el 19 de octubre. Bajo su dirección técnica se revisa el proyecto inicialmente propuesto realizando varias mejoras (19). Las cuatro barras exteriores de las pilas se transforman en cajones huecos, con aperturas para permitir la conexión de las diagonales; esta solución será posteriormente utilizada en la Torre Eiffel.

Seyrig, por otro lado, logró vencer a Eiffel en el concurso de un segundo puente en la ciudad de Oporto en 1880 (40). Se trata del Puente Luis I (Figura 3), en cuyo proyecto volvió a innovar presentando un arco de doble curvatura, pero aumentando el canto hacia los apoyos. En este caso, el arco debía soportar un doble tablero (inferior y superior), y su diseño consiguió reducir la sección de las pilastras sobre el arco y de los tirantes bajo el mismo. El efecto estético es, otra vez, novedoso frente a la propuesta de Eiffel y Cíe (un clon del Maria
Pia). Vuelve a otorgar un valor especial a los elementos principales: cuerda, tangente y arco. La solución recuerda conceptualmente a las de Othmar Ammann en los puentes Hell Gate (1916) y Bayonne (1931) (ambos con forma de tímpano), otras de las obras destacadas por el profesor Billington (39).

\subsection{La estatua y la torre}

La compañía de Eiffel también fue la encargada de diseñar y construir el entramado metálico del interior de la Estatua de la Libertad. Fue ofrecida por Francia a los Estados Unidos para celebrar el centenario de su independencia. Ejecutada íntegramente entre 1881 y 1884 en unos talleres de París, los cálculos fueron llevados a cabo por el propio Koechlin (19), mostrando en su concepción formal y simbólica la estructura de la futura Torre (Figura 6).

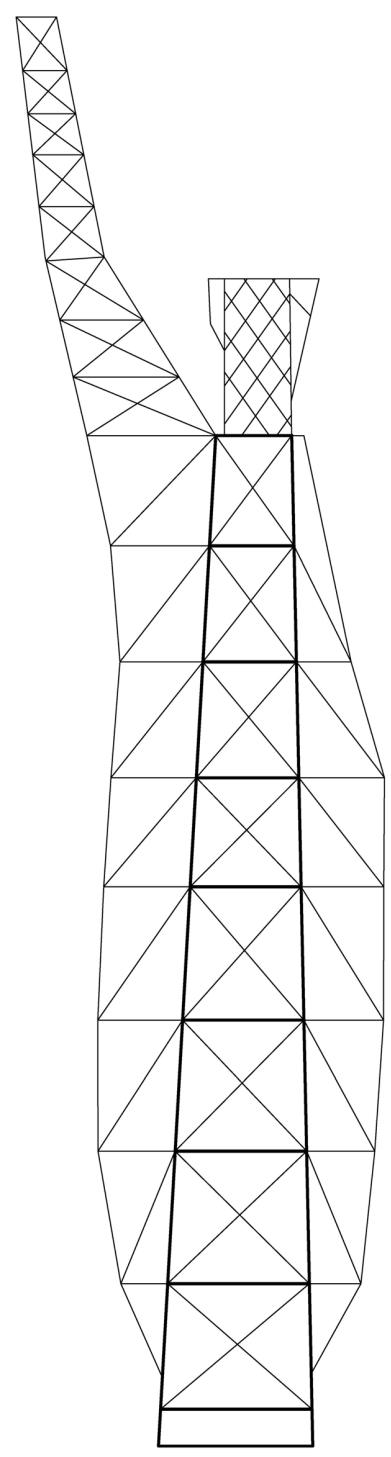

Figura 6. Estatua de la Libertad (Nueva York, 1886). La estructura interna ideada por Koechlin no deja de ser un gigantesco pilono.

Y finalmente la Torre Eiffel. A lo largo de los párrafos anteriores se ha podido comprobar una secuencia lógica en la evolución de las ideas e inquietudes de los técnicos, sobre todo los responsables de la oficina de cálculo de la compañía Eiffel et Cíe y alguno de sus contactos externos. Con los escritos de Nördling empezaba a tomarse conciencia de la especial im- 
portancia del viento en las pilas de gran altura. Y las formas curvas llegaron a su máxima expresión artística en el Puente Maria Pia de Seyrig. Noguier también participa en la construcción de éste. Seyrig logra sorprender una vez más en el de Luis I. Koechlin aprovecha la experiencia acumulada e introduce nuevas ideas y ventajas constructivas en el de Garabit y en la Estatua de la Libertad...

Volvemos al punto de partida, la idea de la torre. Como se apuntaba, fue tras una reunión entre los ingenieros Émile Noguier y Maurice Koechlin (en el año 1884) cuando se empezó a concebir la posibilidad de llegar al reto de los $300 \mathrm{~m}$. En tan sólo dos meses, la habilidad de Koechlin para los cálculos le permitió obtener un primer croquis aproximado de la forma (fechado el 6 de junio de 1884). Y ésta que no era otra que la propia evolución de las ya experimentadas con anterioridad. Cada vez que se había tenido en cuenta de manera racional el efecto del viento, surgían las líneas curvas como adaptación a sus exigencias resistentes. Una vista en planta de cualquiera de los mencionados viaductos sobre el Duero permite obtener una geometría similar a la deducida para la Torre.

La aportación más llamativa de Koechlin la describió el propio Eiffel en su escrito «La Tour de trois cent metres» (1900). A diferencia de las pilas de un puente, en una torre de gran altura no existen los esfuerzos adicionales procedentes de las reacciones horizontales del tablero. Aislando el problema, puede estudiarse una forma curva que evite la necesidad de una celosía interior que reparta estas fuerzas adicionales que ahora no aparecen el cálculo. De esta manera, Koechlin dedujo en muy poco tiempo que podían eliminarse las diagonales interiores. Para ello, bastaba adoptar las siguientes condiciones:

- Primero, que las resultantes de esfuerzos en cada una de las cuatro pilas que conforman el contorno fuesen tangentes a la propia curva.

- Segundo, que la fuerza equivalente al efecto del viento en cualquier porción que se tomara de la torre estuviera aplicada en el mismo punto que la suma de las resultantes que aparecían en cada una de las pilas.

Con esta hipótesis, que proporciona una determinada curvatura de las pilas en dirección vertical, las dos resultantes (viento y pilas) están aplicadas sobre un mismo punto para cada trozo de torre que se ponga en equilibrio. Esto es, no aparecen momentos adicionales, ya que este equilibrio se reduce a una suma de fuerzas. Aunque se coloquen diagonales en el interior, los esfuerzos sobre éstas serán nulos para asegurar el equilibrio de momentos. Koechlin, pudo eliminar estas barras interiores consiguiendo un doble efecto. En primer lugar, reducía la resistencia al viento al disminuir la superficie expuesta. Y en segundo lugar, limpiaba la forma del conjunto, obteniendo un efecto estético hasta ese momento no experimentado y que forma parte del éxito de su integración. Allen y Zalewski mostraron una visión esquemática pero muy didáctica de este método gráfico (42) (Figura 7).

Nuevamente, un estudio racional de la forma resistente en función de las cargas que deben soportarse determina una solución óptima, asegurando la resistencia con un cálculo en coherencia a estas hipótesis básicas y simples. Y además la estética final del conjunto sorprende por su transparencia, limpieza e innovación. Los resultados, y su aceptación tras el paso del tiempo, resultan innegables. Y sus objetivos ampliamente cumplidos. Una disposición tan sorprendente que todavía en nuestros días no pasa desapercibida para cualquier espectador por su sencillez, la adecuada jerarquía estructural, el rigor de su plan de construcción, y su monumentalidad absoluta.

Todo ello se completó con un delicado estudio de los detalles constructivos y aportes estéticos que la arquitectura puede añadir a lo que la forma consigue por sí sola (Figura 1). Quizá fue ésta la gran aportación de Eiffel. Su experiencia acumulada le había permitido discernir en qué aspectos del diseño el metal ofrecía características intrínsecas que no hacía falta matizar, sino que derivaban de la honestidad de las formas estructurales. Y por otra parte, era consciente de que una estructura de grandes dimensiones podía ser estéticamente disfrutada a diferentes escalas de observación:

- La escala de conjunto, que debía integrarse en el paisaje anterior y su futura evolución (en este caso, un entramado urbano de gran riqueza artística en sus edificios).

- La forma de la estructura aislada, coherente con las cargas que debían soportarse.

- La eliminación de elementos superfluos o superabundantes, resultado directo y solución única de las interacciones

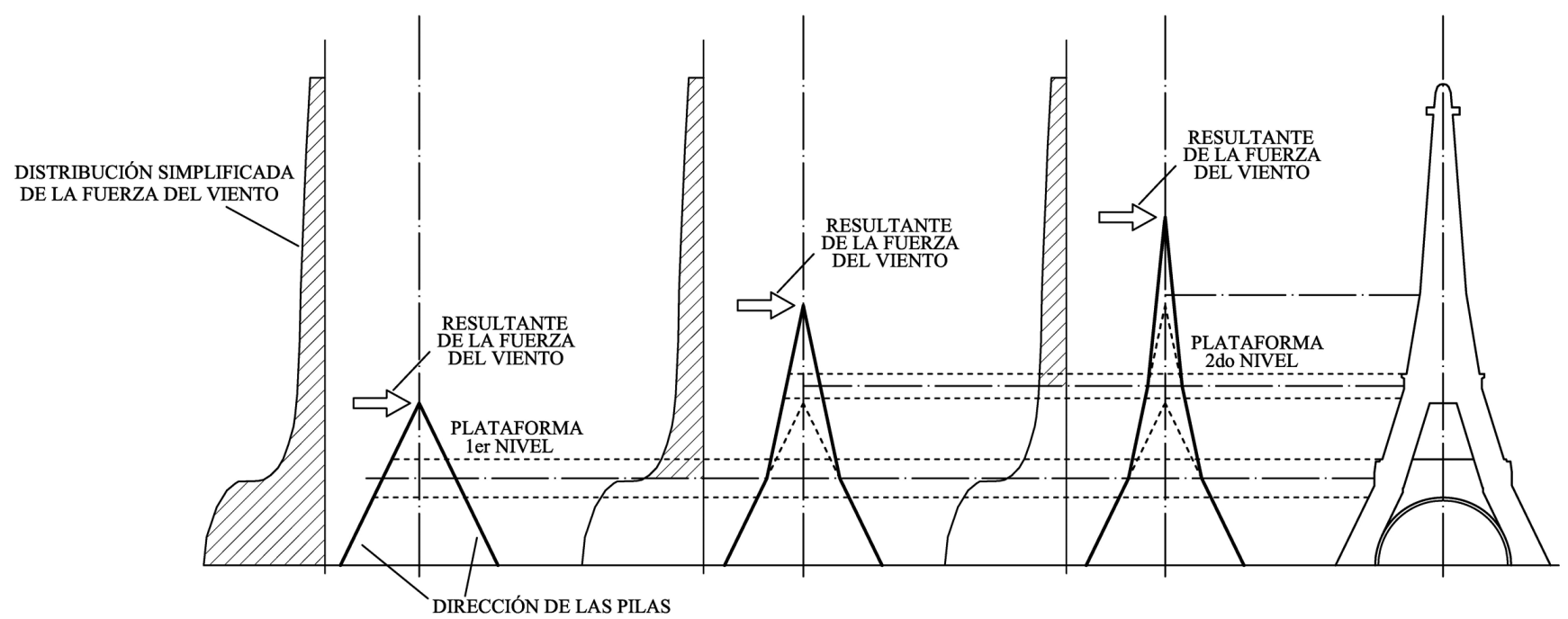

Figura 7. Torre Eiffel (París, 1889). Croquis del diseño de Koechlin. Adaptado de Allen y Zalewski (2009). 
entre las cargas y la forma (como Koechlin obtuvo de manera magistral en su torre sin diagonales).

- La jerarquía estructural, introduciendo entramados y piezas compuestas que acentuaban la transparencia y reducían los esfuerzos de viento, pero resaltaban la propia riqueza de la forma.

- La revisión final de los detalles y nuevos elementos artísticos coherentes con las formas deducidas, sin restarles personalidad.

En este último punto Eiffel demostró su genialidad. Consiguió poner de acuerdo la tecnología y el arte. Supo llamar la atención de los mejores colaboradores que pudo encontrar. Koechlin, un magnífico calculista con ideas sorprendentes, y heredero de las ideas estéticas que Seyrig había puesto de manifiesto en sus construcciones anteriores tal como puede apreciarse desde una perspectiva adecuada en el Puente de Maria Pia (Figura 8). Y en contrapunto, un arquitecto ávido de dotar al metal de todas las posibilidades que ofrecía para ligarle al rigor de las bellas artes.

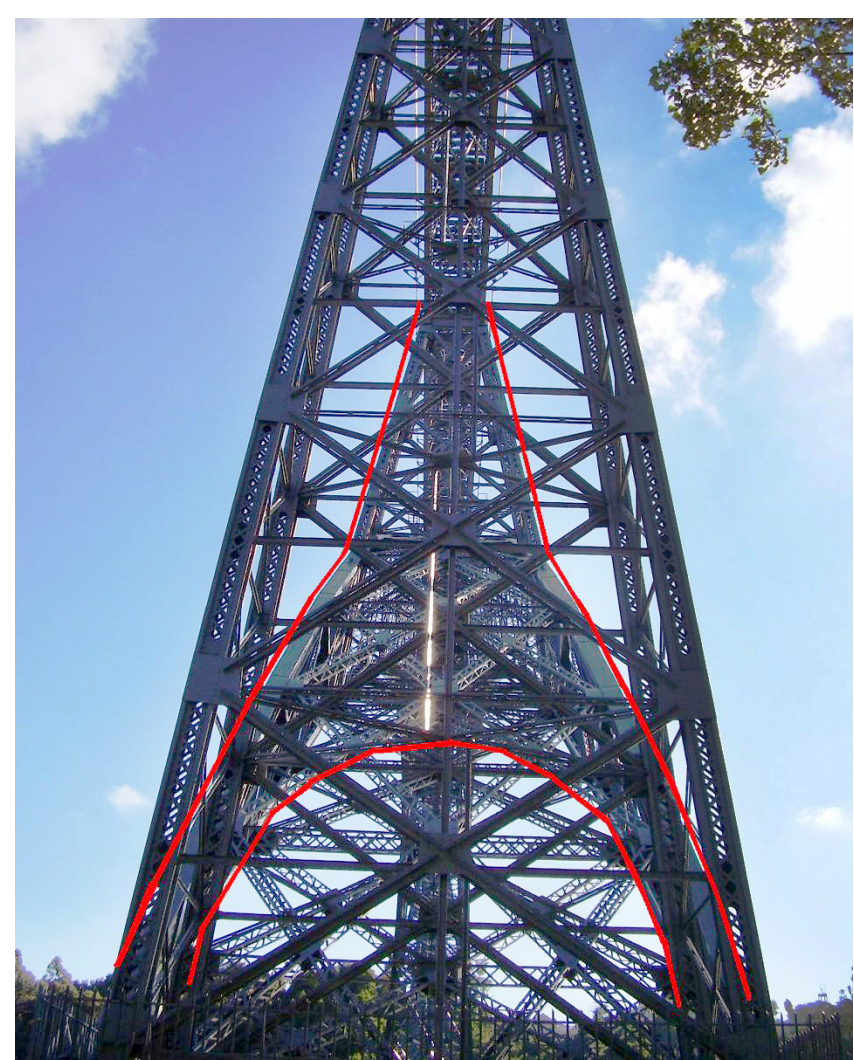

Figura 8. Puente de Maria Pia (Oporto, 1877, fotografía de los autores). El contorno de la Torre Eiffel se esconde en una vista lateral del puente, como un ensayo previo de la forma para la misma acción del viento.
Stephen Sauvestre, un arquitecto con el que trabajó en diferentes ocasiones, fue el encargado de revisar el diseño inicial (19). A través del arco, una forma pura, conectó los diferentes elementos básicos de la estructura para dotarles una continuidad global a diferentes alturas. Estudió la manera de, además, dotar a la torre de los espacios funcionales interiores. Plataformas y miradores se incluyeron en los planos. Se cambió la inclinación de las pilas hasta la primera plataforma, haciéndolas rectas, y sin que esto distorsionara los criterios iniciales.

Incorporó a las líneas básicas infinidad de detalles visibles desde posiciones más cercanas, aprovechando las propias posibilidades del hierro como material. Introdujo elementos casi esculturales ya experimentados en otras edificaciones anteriores, como balaustradas, o filigranas de inspiraciones vegetales. También puede identificarse la jerarquía de formas, en este caso arquitectónica y no estructural: celosías sobre celosías de mayor escala, y arcos que conforman otros arcos de mayor luz. La combinación de detalles a pequeña escala con el diseño global logra un efecto inmejorable.

\section{CONCLUSIÓN}

Hasta el momento, los esfuerzos por definir la geometría de la torre Eiffel se han basado en criterios estrictamente mecánicos y matemáticos. En este artículo se ha propuesto la comparación de otras estructuras usando la perspectiva histórica y analizando su evolución lógica, sobre todo entre los ingenieros y colaboradores cercanos a Eiffel. Se adoptan los criterios propuestos por el profesor Billington en su idea de arte estructural (3), y se asumen para proceder al estudio comparativo. La forma debe de ser entendida como suma de varios saltos tecnológicos que comienzan con las pilas de los puentes y su anchura variable hasta el apoyo. Posteriormente Nördling introduce los abocinados en varios viaductos franceses (1867-1869), y Seyrig aprovecha estas experiencias para introducir la acción del viento como variable crítica en la definición de las formas en estructuras de gran altura. La curvatura surge como característica más importante, siendo el Puente de María Pía (Oporto, 1877) uno de los mejores ejemplos. Noguier y Koechlin asumen todo este conocimiento y lo aprovechan con las nuevas herramientas de cálculo. Seyrig, alejado de Eiffel, vuelve a innovar en el Puente Luis I (Oporto, 1885). La estructura de la Estatua de la Libertad (1886) es el referente simbólico y formal más cercano a la Torre Eiffel (1889), que finalmente es diseñada con una acertada combinación de ingeniería y arquitectura. Se reconocen en el artículo los méritos atribuibles a cada una de las partes. Por un lado los diseñadores, y por otra, la impecable dirección y supervisión de Eiffel, que logró aunar de manera consciente lo mejor de las artes y la técnica de su momento. El resultado es uno de los mejores ejemplos de arte estructural, cuyo simbolismo ha sobrepasado incluso las expectativas de sus propios creadores.

\section{REFERENCIAS}

(1) Holgate, A. (1986). The Art in Structural Design. Oxford: Oxford University Press.

(2) Addis, B. (1994). The Art of the Structural Engineer. Londres: Artemis.

(3) Billington, D. P. (1983). The tower an the bridge. Princeton (NJ): Princeton University Press.

(4) Billington, D. P. (1978). Robert Maillart's Bridges: The Art of Engineering. Princeton (NJ): Princeton University Press.

(5) Billington, D. P. (2003). The Art of Structural Design: A Swiss Legacy. Princeton (NJ): Princeton University Press.

(6) Serrano-López, R., Manso, J. M., Martínez-Martínez, J. A., Payo, R. J. (2012). Los «sentidos» estéticos del patrimonio: la construcción metálica antes del desarrollo de la soldadura. Ejemplos en Burgos y Palencia (España). Informes de la Construcción, 64(528): 457-470, doi: http://dx.doi.org/10.3989/ic.11.108 
(7) Billington, D. P., Gottemoeller, F. (2000). Bridge Aesthetics - Structural Art. In W.-F. Chen y L. Duan (Eds.), Bridge Engineering Handbook. Boca Raton: CRC Press.

(8) Ruskin, J. (1849). The Seven Lamps of Architecture. Nueva York: John Wiley.

(9) Loos, A. (1972). Ornamento y delito y otros escritos. Barcelona: Gustavo-Gili.

(10) Aguiló, M. (2010). Intervención en el coloquio sobre «Arte estructural en ingeniería y arquitectura». http://www.fundacionmiguelaguilo.org/2010/11/arte-estructural-en-ingenieria-y-arquitectura/, fecha de consulta 08/04/2013.

(11) Jones, P. (2006). Ove Arup: Master Builder of the Twentieth Century. London: Yale University Press.

(12) Cassinello, P., Schlaich, M., Torroja, J. A. (2010). Félix Candela. En memoria (1910-1997). Del cascarón de hormigón a las estructuras ligeras del s. XXI. Informes de la Construcción, 62(519): 5-26, doi: http://dx.doi.org/10.3989/ic.10.040.

(13) Azagra, D., Bernabeu, A. (2012). La estructura de las formas libres. Informes de la Construcción, 64(526): 133-142, doi: http://dx.doi.org/10.3989/ic.11.015.

(14) Rabasco, P. (2011). El sistema Ctesiphonte. Evolución de la estructura catenaria. Informes de la Construcción, 63(522): 43-52, doi: http://dx.doi.org/10.3989/ic.10.009

(15) Gallant, J. (2002). The shape of the Eiffel Tower. American Journal of Physics, 70(2): 160-162, doi: http://dx.doi. org/10.1119/1.1417530.

(16) Weidman, P., Pinelis, I. (2004). Model equations for the Eiffel Tower profile: historical perspective and new results. Comptes Rendus Mecanique, 332(7): 571-584, doi: http://dx.doi.org/10.1016/j.crme.2004.02.021.

(17) Lakes, R. (1993). Materials with structural hierarchy. Nature, 361: 511-515, doi: http://dx.doi.org/10.1038/361511ao.

(18) Meenakshi, M., Ananthasuresh, G. K. (2009). Gustave eiffel and his Optimal Strcutures. Resonance, 14(9): 849-865, doi: http://dx.doi.org/10.1007/s12045-009-0081-x.

(19) Lemoine, B. (2002). Gustave Eiffel. Madrid: Ediciones Akal.

(20) Lemoine, B. (1995). L'Entreprise Eiffel. Historie, économie et société, 14(14-2): 273-285, doi: http://dx.doi.org/10.3406/ hes.1995.1773.

(21) Ellis, J. S. (1986). Gustave Eiffel: a biographical sketch. J. Struct. Eng. 112(6): 1404-1412, doi: http://dx.doi.org/10.1061/ (ASCE)0733-9445(1986)112:6(1404).

(22) Barjot, D. (1987). L'innovation dans les travaux publics (XlXe-XXe siècles). Une réponse des firmes au défi de la demande publique ? Histoire, économie et société, 6(6-2): 209-231, doi: http://dx.doi.org/10.3406/hes.1987.1447.

(23) Kliczkowski, H. (2003). Gustave Alexandre Eiffel. Barcelona (Spain): Loft Publications.

(24) González, E., Sánchez, J. I. (1995). Presencia de la Escuela de Eiffel - Seyrig en el Duero. Los puentes del ferrocarril de Ariza. Anales de arquitectura, 6: 33-54.

(25) Société des Ingéniuers Civils de France. (1906). Annuaire de 1906. Paris, France: Société des Ingéniuers Civils de France.

(26) Los diferentes motores mecánicos para tranvías y ferrocarriles secundarios. (1895). Revista ilustrada de vías férreas, (25 Enero): 21,

(27) Escudier, J.-L. (1995). Itinéraire d'un entrepreneur de travaux publics éclectique: Edmond Bartissol (1841-1916). Histoire, économie et société, 14(14-2): 229-251, doi: http://dx.doi.org/10.3406/hes.1995.1771.

(28) Martínez, E. (1891). Zamora. Puente de hierro sobre el río Duero en el ferrocarril de Malpartida a Astorga. La ilustración española y americana, Año XXXV(XXII): 363-372.

(29) Corrimiento de los puentes. (1933, octubre). Heraldo de Zamora, (7).

(30) AFAS. (1882). Compte Rendu de la $10^{a}$ Session. Alger, 1881. Paris, France: Association francaise pour l'avancement des sciences.

(31) Guillet, L. (1929). Cent ans de la vie de l'Ecole Centrale des Arts et Manufactures- 1829-1929. Paris (France): Editions artistiques de Paris, M. de Brunoff.

(32) Serna, J., Rui-Wamba, J. (2006). Los puentes del Tren. Madrid (Spain): Fundación Esteyco.

(33) Fernandez, L. (1999). Tierra sobre el agua. Visión Histórica Universal de los Puentes. Madrid (Spain): Colegio de Ingenieros de Caminos, Canales y Puertos.

(34) Nördling, W. (1864). Mémoire sur les piles en charpente métallique des grands viaducs. Annales des Pont et Chaussées, $\operatorname{VIII}(83): 1-126$,

(35) Gaag, C. R., Lewis, P. R. (2011). The rise and fall of cast iron in Victorian structures - A case study review. Eng Fail Anal, 18: 1963-1980, doi: http://dx.doi.org/10.1016/j.engfailanal.2011.07.013.

(36) Santos, J. C. (1989). O Palácio de Cristal e Arquitectura de Ferro no Porto em Meados do Século XIX. Porto (Portugal): Fundação Engenheiro António de Almeida.

(37) Sousa-Cruz, P. J., Lopes-Cordeiro, J. M. (2003). Audacious and elegant 19th century Porto bridges. Pract Periodical Struct Des Construct, 8(4): 217-225, doi: http://dx.doi.org/10.1061/(ASCE)1084-0680(2003)8:4(217).

(38) Thrall, A. P., Billington, D. P., Bréa, K. L. (2012). The Maria Pia Bridge: A major work of structural art. Eng Struct, 40: 479-486, doi: http://dx.doi.org/10.1016/j.engstruct.2012.02.032.

(39) Thrall, A. P., Billington, D. P. (2009). A study of the evolution of arch forms: Eiffel's Maria Pia Bridge and Ammann's Bayonne Bridge. En K. M. Mahmoud (Ed.), Safety and Reliability of Bridge Structures (pp. 421-428). London (UK): Taylor \& Francis - CRC Press.

(40) Sousa-Cruz, P. J., Lopes-Cordeiro, J. M. (2001). As pontes do Porto. Porto (Portugal): Livraria Civilizaçao.

(41) Kurrer, K. E. (2008). The History of the Theory of Structures. Berlin (Germany): Ernst \& Sohn.

(42) Allen, E., Zalewski, W. (2009). Form and forces: designing efficient, expressive structures. Hoboken, NJ: John Wiley \& Sons. 